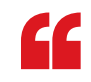

binding of

infected cells to the endothelia of the brain, heart, lung and bone marrow was mediated by EPCR.

PARASITE BIOLOGY

\title{
EPCR unlocks severe malaria
}

Sequestration of Plasmodium falciparum-infected erythrocytes in the microvasculature is key to the pathogenesis of severe malaria and involves interactions between $P$. falciparum erythrocyte membrane protein 1 (PfEMP1) and receptors on the endothelial lining. Now, Turner et al. identify endothelial protein $C$ receptor (EPCR) as the specific receptor for PfEMP1 variants that are associated with severe childhood malaria.

The P. falciparum genome contains about 60 var genes encoding distinct PfEMP1 variants that are expressed on the surface of infected erythrocytes and attach to various receptors on the endothelial lining. In severe childhood malaria, PfEMP1 subtypes containing domain cassette 8 (DC8) and DC13 predominate, but the endothelial receptor that these ligands bind to was unknown. To identify the receptor for DC8-containing PfEMP1, the authors purified a recombinant version of this protein and screened it against a library of 2,505 human plasma membrane proteins using microarrays. The screen identified EPCR as a potential binding partner, and enzyme-linked immunosorbent assays mapped EPCR binding to the CIDRa1 domains of DC8 and DC13.

The authors then assessed whether infected erythrocytes expressing native DC8-containing PfEMP1 could bind EPCR on endothelial cells. They found that binding of infected cells to the endothelia of the brain, heart, lung and bone marrow was mediated by EPCR. Next, the authors compared the binding phenotypes of parasites isolated from patients with severe, uncomplicated or mild malaria. Binding to EPCR was shown to be significantly higher among parasites from patients with severe malaria than among parasites associated with uncomplicated or mild forms of the disease, demonstrating that EPCR binding is a common phenotype of parasites that cause severe malaria.

The pathology of severe malaria seems to be linked to an exaggerated inflammatory response. Interestingly, EPCR is typically involved in promoting cytoprotection and anti-inflammatory signalling, owing to interactions with activated protein C (APC). Surface plasmon resonance analysis revealed that

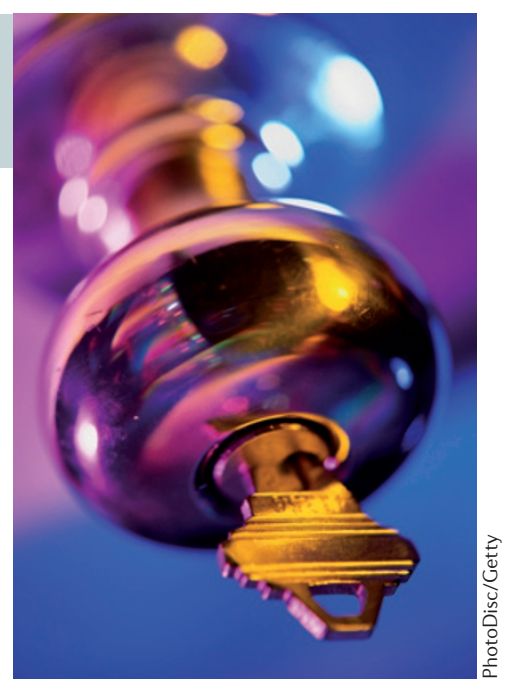

pre-incubation of EPCR with either the DC8 CIDRa1.1 domain or the DC13 CIDRa1.4 domain inhibited binding of APC, indicating that these PfEMP1 domains compete for the same binding site as APC.

These findings identify EPCR as a specific receptor for $P$. falciparuminfected erthrocytes and suggest that binding to this receptor interferes with its anti-inflammatory role, possibly contributing to the pathology of severe malaria.

Christina Tobin Kåhrström

ORIGINAL RESEARCH PAPER Turner, L. et al.

Severe malaria is associated with parasite binding to endothelial protein C receptor. Nature 5 Jun 2013 (doi:10.1038/nature12216) 